\title{
Approximation of Alcohol-Based Hand Sanitizer Volume Using a Toothpaste Cap \\ (1)
}

\author{
Mohit Kumar Gupta, BBA; Shari R. Lipner, MD, PhD
}

Physicians and the general public may be underusing alcohol-based hand sanitizers based on the false perception that they are damaging to the skin. We describe the use of a screw-top toothpaste cap to approximate the necessary volume of alcohol-based sanitizer for adequate hand disinfection.

Cutis. 2020;106:133.

\section{Practice Gap}

The Centers for Disease Control and Prevention recommends handwashing with soap and water or using alcohol-based hand sanitizers to prevent transmission of coronavirus disease 2019. Five steps are delineated for effective handwashing: wetting, lathering, scrubbing, rinsing, and drying. Although alcohol-based sanitizers may be perceived as more damaging to the skin, they are less likely to cause dermatitis than handwashing with soap and water. ${ }^{1}$ Instructions are precise for handwashing, while there are no recommendations for effective use of alcohol-based hand sanitizers. A common inquiry regarding alcohol-based hand sanitizers is the volume needed for efficacy without causing skin irritation.

\section{The Technique}

Approximately $1 \mathrm{~mL}$ of alcohol-based hand sanitizer is recommended by some manufacturers. However, abundant evidence refutes this recommendation, including a study that tested the microbial efficacy of alcohol-based sanitizers by volume. A volume of $2 \mathrm{~mL}$ was necessary to achieve the $2.0 \mathrm{log}$ reduction of contaminants as required by the US Food and Drug Administration for antimicrobial efficacy. ${ }^{2}$ The precise measurement of hand sanitizer using a calibrated syringe before each use is impractical. Thus, we recommend using a screw-top toothpaste cap to assist in approximating the necessary volume (Figure). The cap holds approximately $1 \mathrm{~mL}$ of liquid as measured using a syringe; therefore, 2 caps filled with sanitizer should be used.

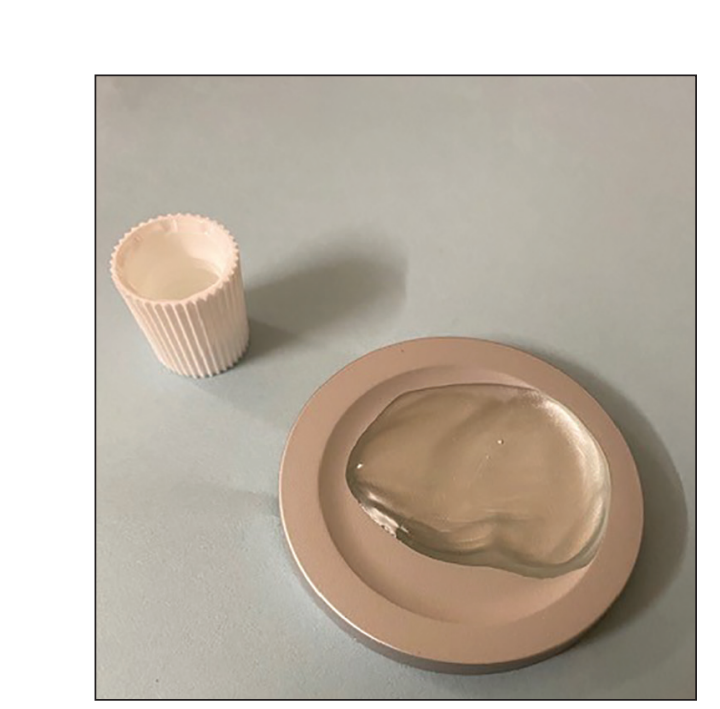

Visual approximation for the appropriate volume $(2 \mathrm{~mL})$ of an alcoholbased hand sanitizer needed for disinfection as measured by completely filling the screw-top cap of a toothpaste tube twice.

\section{Practice Implications}

The general public may be underutilizing hand sanitizer due to fear of excessive skin irritation or supply shortages, which will reduce efficacy. Patients and physicians can use this simple visual approximation to ensure adequate use of hand sanitizer volume.

\section{REFERENCES}

1. Stutz N, Becker D, Jappe U, et al. Nurses' perceptions of the benefits and adverse effects of hand disinfection: alcohol-based hand rubs vs. hygienic handwashing: a multicentre questionnaire study with additional patch testing by the German Contact Dermatitis Research Group. Br J Dermatol. 2009;160:565-572.

2. Kampf G, Ruselack S, Eggerstedt S, et al. Less and less-influence of volume on hand coverage and bactericidal efficacy in hand disinfection. BMC Infect Dis. 2013;13:472.

Mr. Gupta is from the State University of New York Downstate College of Medicine, Brooklyn. Dr. Lipner is from the Department of Dermatology, Weill Cornell Medicine, New York, New York.

The authors report no conflict of interest.

Correspondence: Shari R. Lipner, MD, PhD, 1305 York Ave, New York, NY 10021 (shl9032@med.cornell.edu).

doi:10.12788/cutis.0045 